\title{
Satellite to Satellite Visibility
}

\author{
M.A. Sharaf ${ }^{1}$, Z.M. Hayman ${ }^{2, *}$ and M.E. Awad ${ }^{2}$ \\ ${ }^{I}$ Department of Astronomy, Faculty of Science, King Abdul Aziz University, Jeddah, Saudi Arabia \\ ${ }^{2}$ Department of Astronomy, Faculty of Science, Cairo University, Giza, Egypt
}

\begin{abstract}
In this paper, general analytical and computational technique for satellite-to-satellite visibility will be established. The developments are general in the sense that the visibility conditions can be used whatever the types of the satellite orbits may be. Numerical applications are also included.
\end{abstract}

Keywords: Satellite, visibility, orbit, numerical solution.

\section{INTRODUCTION}

The use of satellites in communications systems is very much a fact of everyday life, as it is evidenced by many homes which are equipped with many antennas, or dishes used for the reception of satellite television.

Satellites offer a number of features not readily available with other means of communications. As very large areas of the Earth are visible from a satellite, a satellite can form the starting point of a communications net linking together with many users simultaneously, users who may be widely separated geographically [1].

Communication satellites account for more than $80 \%$ of the total number of satellites in operations. This is one of the most widely exploited applications of satellites. On the other hand, there are many uses of the communication satellites, e.g., in Earth's sciences solar physics, astronomy, astrophysics and military purposes [2].

Extensive recovery experiments based on real CHAMP GPS SST observations have shown that the concept of estimating non- gravitational accelerations from GPS observation is feasible [3] and the full potential of GPS-SST in gravity field recovery becomes evident if employed on a very low - flying satellite has been demonstrated [4-6].

Any application which attempts to use communication satellites requires effective maneuvering to ensure an accurate result.

To this end and also to cope with these very important applications of satellites in communication as well as in space warfare, we establish (from astrodynamical point of view) in the present paper general analytical and computational technique for one of the most important problems of space communications, of satellite-to-satellite visibility.

The developments are general in the sense that visibility conditions will be established, whatever the types of the satellite orbits may be.

*Address correspondence to this author at the Dept. of Astronomy and Meteorology, Fac. of Science, Cairo University, Egypt;

Tel: +2010-1001644; Fax: +202-35717026;

E-mails: zmhayman@gmail.com, zmhayman@yahoo.com

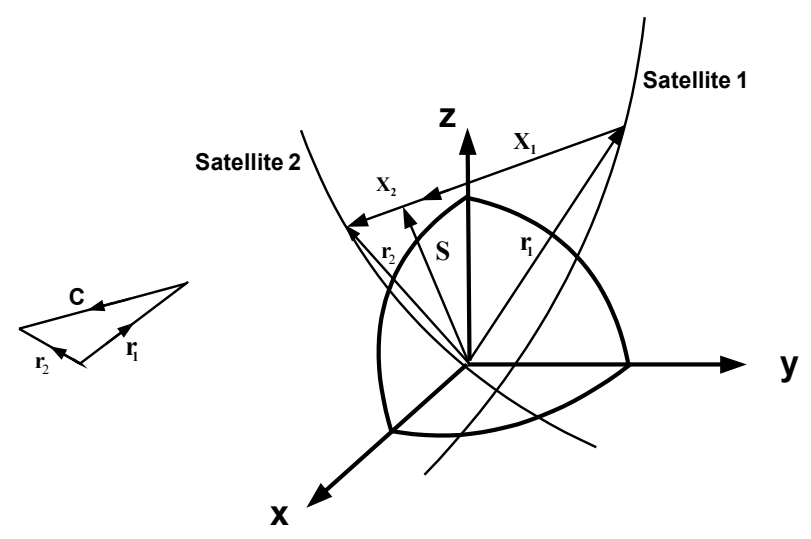

Fig. (1). Relative rise-set geometry.

\section{DEVELOPMENT OF THE RISE-SET FUNCTION}

Mutually visible satellites are defined in this section as two satellites that can maintain direct line of sight between each other for a certain length of time. Actually, the analysis to be developed is primarily concerned with the rise and set time of a given satellite with respect to another, that is, the time of loss or gain of direct line of sight.

\subsection{Relative Rise-Set Geometry}

Consider the geometry defined in Fig. (1). As illustrated, satellites 1 and 2 are in a state of relative rise or set. Indeed, if the vector $S$, which emanates from the dynamical center of the Earth, had a magnitude equal to or less than the radius of the Earth and if it were perpendicular to $C$, the chord length between the satellites, it is evident that the satellites would not have direct line-of-sight-communication. Owing to atmospheric interference, however, a realistic analysis would let the magnitude of $S$ be slightly larger than $a_{e}$, the radius of the Earth. Let $\triangle$ be the thickness of the atmosphere or suitable bias factor, it follows that

$S^{2}=\vec{S} \cdot \vec{S}=\left(a_{e}+\Delta\right)^{2}$

\subsection{Analytical Expression of the Relative Rise-Set Function}

Examination of Fig. (1) allows the two fundamental vector closure equations to be written as 


$$
\begin{aligned}
& \vec{r}_{2}=\vec{S}+\bar{\chi}_{2} \\
& \vec{r}_{1}=\vec{S}-\bar{\chi}_{1}
\end{aligned}
$$

Where $\vec{r}_{i} ; \mathrm{i}=1,2$ are the position vectors of the satellites and $\bar{\chi}_{i} ; \mathrm{i}=1,2$ are the two unknown vectors.

At relative rise and set of satellite 1 with respective to satellite 2, we have

$\vec{S} \cdot \bar{\chi}_{1}=\vec{S} \cdot \bar{\chi}_{2}=0$

Then, from the figure

$$
C=\sqrt{\left(\left(\vec{r}_{2}-\vec{r}_{1}\right),\left(\vec{r}_{2}-\vec{r}_{1}\right)\right)}=\sqrt{r_{2}^{2}+r_{1}^{2}-2 \alpha} .
$$

Where

$$
\alpha=\vec{r}_{1} \cdot \vec{r}_{2} .
$$

It is then possible to obtain an analytical expression for the rise and set function as

$$
R=\left(\vec{r}_{1} \cdot \vec{r}_{2}\right)^{2}-r_{2}^{2} r_{1}^{2}+\left(r_{2}^{2}+r_{1}^{2}\right) S^{2}-2 S^{2}\left(\vec{r}_{1} \cdot \vec{r}_{2}\right)
$$

Where $S$ is obtained from Equation (1).

\subsection{Visibility using Equation (6)}

The rise-set function defined in Equation (6) can be used to predict explicitly whether or not satellites are visible to one another .The sign of $\mathrm{R}$ associated with visibility can be obtained by constructing a case in which direct line -ofsight visibility is impossibile, consequently we can get the rule that:

- Negative value of $R \Rightarrow$ Direct line-of-sight communication

- Positive value of $R \Rightarrow$ non-visibility

\subsection{Reduction of Rise-Set Function to Two Parameters Function}

In terms of the orbital eccentricity e, semi-parameter $p$ and the true anomaly $\mathrm{f}$, the equation of each orbit can be expressed by the relation

$$
r_{i}=\frac{p_{i}}{1+e_{i} \cos \left(f_{i}\right)}, \mathrm{i}=1,2
$$

Also we have

$$
\vec{r}_{\mathrm{i}}=\hat{\mathrm{I}}_{\mathrm{i}} \vec{P}_{\mathrm{i}}+C_{\mathrm{i}} \vec{Q}_{\mathrm{i}}, \mathrm{i}=1,2
$$

Where

$$
\begin{aligned}
& \hat{\mathrm{I}}_{\mathrm{i}}=r_{\mathrm{i}} \cos \left(f_{\mathrm{i}}\right) \\
& \eta_{\mathrm{i}}=r_{\mathrm{i}} \sin \left(f_{\mathrm{i}}\right)
\end{aligned}
$$

The standard orientation vectors $P$ and $Q$, where $P$ is unit vector from the dynamical centre which points at perigee of the orbit and $Q$ is advanced to $P$ by a right angle in the plane and in the direction of motion that is

$$
\begin{aligned}
& P_{x i}=\cos \left(\omega_{i}\right) \cos \left(\Omega_{i}\right)-\sin \left(\omega_{i}\right) \sin \left(\Omega_{i}\right) \cos \left(I_{i}\right) \\
& P_{y i}=\cos \left(\omega_{i}\right) \sin \left(\Omega_{i}\right)+\sin \left(\omega_{i}\right) \cos \left(\Omega_{i}\right) \cos \left(I_{i}\right) \\
& P_{z i}=\sin \left(\omega_{i}\right) \sin \left(I_{i}\right)
\end{aligned}
$$

$Q_{x i}=P_{x i}\left(I, \omega+90^{\circ}, \Omega\right)=-\sin \left(\omega_{i}\right) \cos \left(\Omega_{i}\right)+\cos \left(\omega_{i}\right) \sin \left(\Omega_{i}\right) \cos \left(I_{i}\right)(11.4)$

$Q_{y i}=P_{y i}\left(I, \omega+90^{\circ}, \Omega\right)=-\sin \left(\omega_{i}\right) \sin \left(\Omega_{i}\right)+\cos \left(\omega_{i}\right) \cos \left(\Omega_{i}\right) \cos \left(I_{i}\right)(11.5)$

$Q_{z i}=P_{z i}\left(I, \omega+90^{\circ}, \Omega\right)=\cos \left(\omega_{i}\right) \sin \left(I_{i}\right)$

Where $I, \omega$ and $\Omega$ are the inclination, longitude of per center and longitude of the node, respectively.

Now

$$
\begin{aligned}
\left\langle\vec{r}_{1}, \vec{r}_{2}\right\rangle & =\left\langle\left(\xi_{1} \vec{P}_{1}+\eta_{1} \vec{Q}_{1}\right),\left(\xi_{2} \vec{P}_{2}+\eta_{2} \vec{Q}_{2}\right)\right\rangle \\
& =A_{1} \xi_{1} \xi_{2}+A_{2} \eta_{1} \xi_{2}+A_{3} \eta_{2} \xi_{1}+A_{4} \eta_{1} \eta_{2},
\end{aligned}
$$

Where

$\mathrm{A}_{1}=\left\langle\mathbf{P}_{1}, \mathbf{P}_{2}\right\rangle, \mathrm{A}_{2}=\left\langle\mathbf{Q}_{1}, \mathbf{P}_{2}\right\rangle$

$\mathrm{A}_{3}=\left\langle\mathbf{P}_{1}, \mathbf{Q}_{2}\right\rangle, \mathrm{A}_{4}=\left\langle\mathbf{Q}_{1}, \mathbf{Q}_{2}\right\rangle$

Using Equations (9) and (10) into Equation (12) we get $\left\langle\mathbf{r}_{1}, \mathbf{r}_{2}\right\rangle=\frac{\mathrm{p}_{1} \mathrm{p}_{2} \cos \left(\mathrm{f}_{2}-\mathrm{f}_{1}\right)}{\left(1+\mathrm{e}_{1} \cos \mathrm{f}_{1}\right)\left(1+\mathrm{e}_{2} \cos \mathrm{f}_{2}\right)}\left\{\cos \mathrm{f}_{2}\left(\mathrm{~A}_{1} \cos \mathrm{f}_{1}+\mathrm{A}_{2} \sin \mathrm{f}_{1}\right)+\sin \mathrm{f}_{2}\left(\mathrm{~A}_{3} \cos \mathrm{f}_{1}+\mathrm{A}_{4} \sin \mathrm{f}_{1}\right)\right\}$.

Or,

$$
\left\langle\mathbf{r}_{1}, \mathbf{r}_{2}\right\rangle=\frac{\mathrm{p}_{1} \mathrm{p}_{2}}{\left(1+\mathrm{e}_{1} \cos \mathrm{f}_{1}\right)\left(1+\mathrm{e}_{2} \cos \mathrm{f}_{2}\right)}\left\{\mathrm{D}_{1} \cos \mathrm{f}_{2} \cos \left(\tilde{\mathrm{a}}_{1}-\mathrm{f}_{1}\right)+\mathrm{D}_{2} \operatorname{sinf} \mathrm{f}_{2} \cos \left(\emptyset_{1}-\mathrm{f}_{1}\right)\right\}
$$

Where

$\varnothing_{1}=\tan ^{-1}\left(\frac{\mathrm{A}_{4}}{\mathrm{~A}_{3}}\right) \quad$ and $\quad \tilde{\mathrm{a}}_{1}=\tan ^{-1}\left(\frac{\mathrm{A}_{2}}{\mathrm{~A}_{1}}\right)$

Then Equation (6) becomes

$$
\begin{aligned}
& \mathrm{R}=\mathrm{p}_{1}^{2} \mathrm{p}_{2}^{2}\left\{\mathrm{D}_{1} \cos \mathrm{f}_{2} \cos \left(\tilde{\mathrm{a}}_{1}-\mathrm{f}_{1}\right)+\mathrm{D}_{2} \sin \mathrm{f}_{2} \cos \left(\emptyset_{1}-\mathrm{f}_{1}\right)\right\}^{2}-\mathrm{p}_{1}^{2} \mathrm{p}_{2}^{2}+ \\
& +\left\{\mathrm{p}_{1}^{2}\left(1+\mathrm{e}_{1} \cos \mathrm{f}_{1}\right)^{2}+\mathrm{p}_{2}^{2}\left(1+\mathrm{e}_{2} \cos \mathrm{f}_{2}\right)^{2}\right\} \mathrm{S}^{2}- \\
& 2 \mathrm{~S}^{2} \mathrm{p}_{1} \mathrm{p}_{2}\left\{\mathrm{D}_{1} \cos \mathrm{f}_{2} \cos \left(\tilde{\mathrm{a}}_{1}-\mathrm{f}_{1}\right)+\mathrm{D}_{2} \sin \mathrm{f}_{2} \cos \left(\emptyset_{1}-\mathrm{f}_{1}\right)\right\} \times \\
& \times\left(1+\mathrm{e}_{1} \cos \mathrm{f}_{1}\right)\left(1+\mathrm{e}_{2} \cos \mathrm{f}_{2}\right)
\end{aligned}
$$

If the two satellites are in the same orbital plane we have

$$
\begin{aligned}
& \mathbf{P}_{1}=\mathbf{P}_{2}, \mathbf{Q}_{1}=\mathbf{Q}_{2} \Rightarrow A_{1}=1 \\
& A_{2}=0, A_{3}=0, A_{4}=1 \Rightarrow D_{1}=1, D_{2}=1, \tilde{a}_{1}=0, \varnothing=90^{\circ}
\end{aligned}
$$

\section{COMPUTATIONAL ALGORITHM}

In what follows computational algorithm of the mutual visibility between two satellites will be established whatever the types of their orbits may be:

-Purpose: Mutual visibility between two Earth satellites.

-Input: $\mathrm{a}_{i}$ or $\mathrm{q}_{\mathrm{i}}, \mathrm{e}_{\mathrm{i}}, \mathrm{I}_{\mathrm{i}}, \omega_{\mathrm{i}}, \Omega_{\mathrm{i}}, \mathrm{T}_{\mathrm{i}} \mathrm{i}=1,2 ; \mathrm{S}, \Delta, \mathrm{t}, \mathrm{k}, \mu$.

\section{Computational Sequence:}

$$
\begin{aligned}
& \text { 1- If } \mathbf{E}_{\mathbf{I}}>\mathbf{1} \text { then } \mathbf{N}_{\mathbf{J}}=k \sqrt{\frac{\grave{I}}{-\mathrm{a}_{\mathrm{i}}{ }^{3}}} \text { and } \mathrm{q}_{\mathrm{i}}=\mathrm{a}_{\mathrm{i}}\left(1-\mathrm{e}_{\mathrm{i}}\right) \\
& \text { 2- If } \mathrm{e}_{\mathrm{i}} \cong 1 \text { then } \mathrm{n}_{\mathrm{j}}=k \sqrt{\frac{\mu}{2 q_{i}{ }^{3}}}
\end{aligned}
$$


3- If $\mathrm{e}_{\mathrm{i}}<1$ then $\mathrm{n}_{\mathrm{j}}=\mathrm{k} \sqrt{\frac{\grave{\mathrm{I}}}{\mathrm{a}_{\mathrm{i}}{ }^{3}}}$ and $\mathrm{q}_{\mathrm{i}}=\mathrm{a}_{\mathrm{i}}\left(1-\mathrm{e}_{\mathrm{i}}\right)$

4- $\mathrm{M}_{\mathrm{i}}=\mathrm{n}_{\mathrm{i}}\left(\mathrm{t}-\mathrm{T}_{\mathrm{i}}\right)$.

5 - If $e_{i}<1$ then solve $F_{i}$ from Kepler's equation of hyperbolic orbit using Newton's method and then $\mathrm{f}_{\mathrm{i}}$ as follows

a- let $\left(\mathrm{F}_{\mathrm{i}}\right)_{0}=6 \mathrm{M}_{\mathrm{i}}$

b- $\left(F_{i}\right)_{n+1}=\left(F_{i}\right)_{n}+\frac{M_{i}-e_{i} \sinh \left(F_{i}\right)_{n}+\left(F_{i}\right)_{n}}{e_{i} \cosh \left(F_{i}\right)_{n}-1}$

c- If $\left|\left(F_{i}\right)_{n+1}-\left(F_{i}\right)_{n}\right|>0.00000001$ go to b else $F_{i}=\left(F_{i}\right)_{n+1}$

$\mathrm{d}-\mathrm{f}_{\mathrm{i}}=\tan ^{-1}\left(\frac{-\sinh \left(\mathrm{F}_{\mathrm{i}}\right) \sqrt{\mathrm{e}_{\mathrm{i}}^{2}-1}}{\cosh \left(\mathrm{F}_{\mathrm{i}}\right)-\mathrm{e}_{\mathrm{i}}}\right)$ and end.

6 - If $\mathrm{e}_{\mathrm{i}} \cong 1$ then solve $\mathrm{f}_{\mathrm{i}}$ from Barkar's equation as follows

a-Let $\mathrm{A}_{\mathrm{i}}=3 M_{i} / 2$

$\mathrm{b}-\mathrm{B}_{\mathrm{i}}=\left(\sqrt{A_{i}^{2}+1}+A_{i}\right)^{1 / 3}$

C- $\mathrm{C}_{\mathrm{i}}=B_{i}-\left(1 / B_{i}\right)$

d- $\mathrm{f}_{\mathrm{i}}=2 \tan ^{-1} \mathrm{C}_{i}$

7- If $e_{i}<1$ then solve for $E_{i}$ from Kepler's equation using Newton's method and then

$\mathrm{f}_{\mathrm{i}}$ as follows

a- let $\left(\mathrm{E}_{\mathrm{i}}\right)_{0}=\mathrm{M}_{\mathrm{i}}$

b- $\left(E_{i}\right)_{n+1}=\left(E_{i}\right)_{n}+\frac{M_{i}+e_{i} \sin \left(E_{i}\right)_{n}-\left(E_{i}\right)_{n}}{1-e_{i} \cos \left(E_{i}\right)_{n}}$

c- If $\left|\left(E_{i}\right)_{n+1}-\left(E_{i}\right)_{n}\right|>0.00000001$ go to b else $E_{i}=\left(E_{i}\right)_{n+1}$

$d-f_{i}=\tan ^{-1}\left(\frac{\sin \left(E_{i}\right) \sqrt{1-e_{i}^{2}}}{\cos \left(E_{i}\right)-e_{i}}\right)$ and end.

$8-r_{i}=\frac{\left(1+e_{i}\right) q_{i}}{1+e_{i} \cos f_{i}}$

9-P and Qfrom Equation (11).

10- $\hat{\mathrm{I}}_{\mathrm{i}}=\mathrm{r}_{\mathrm{i}} \cos \left(\mathrm{f}_{\mathrm{i}}\right)$ and $\mathrm{C}_{\mathrm{i}}=\mathrm{r}_{\mathrm{i}} \sin \left(\mathrm{f}_{\mathrm{i}}\right)$.

$11-\mathbf{r}_{i}=\hat{\mathrm{I}}_{\mathrm{i}} \mathbf{P}_{\mathrm{i}}+\mathrm{C}_{\mathrm{i}} \mathbf{Q}_{\mathrm{i}}$.

12-Compute the mutual visibility function $\mathrm{R}$ from Equation (14). Whenever this value is negative, the satellites can see each other at the given time $t$.

13-The algorithm is completed.

\section{CONCLUSION}

We will apply the above algorithm on the seven satellites to get the time and date at which one satellite observes the other.

We will take as an example the following seven satellites. Satellite_1, Satellite_2, Satellite_3 and Satellite_4 are nearly circular, Satellite_5 is elliptical orbit, but Satellite_6 is parabolic orbit and finally Satellite_7 is hyperbolic orbit. The two-line elements and their names of seven satellites are:

\section{Satellite_1}

\section{EGYPTSAT 1}

$131117 \mathrm{U}$ 07012A 08142.74302347.00000033 00000-0 13654-402585

231117098.0526218 .76380007144061 .2019298 .9894 14.6988765758828

\section{Satellite_2}

TRMM

1 25063U 97074A 08141.84184490.00002948 00000-0 41919-4 07792

225063034.9668053 .58650001034271 .1427088 .9226 15.55875272598945

\section{Satellite_3}

GOES 3

1 10953U 78062A 08140.64132336-.00000110 00000-0 10000-3 01137

210953014.2164003 .19680001795336 .4858023 .4617 01.0028002762724

\section{Satellite_4}

NOAA 3

1 06920U 73086A $08141.92603915-.00000030+00000-$ $0+10000-3000067$

206920101.7584171 .94300006223187 .3360172 .7614 12.40289355563642

\section{Satellite_5}

NAVSTAR 46

1 25933U 99055A 08142.14123352.00000019 00000-0 10000-3000126

225933051.0650222 .94390079044032 .8625327 .6958 02.0056810263184

\section{Satellite_6}

Parabola

1 00000U 00000A 08141.53396007.00000000 00000-0 00000-0 000001

200000035.3423067 .8765001 .000253 .7654138 .0987 02.6578654463184

\section{Satellite_7}

Hyp_1

1 00000U 00000A 08141.89332000.00000000 00000-0 00000-0 000001

200000072.8721105 .6746001 .164065 .8757221 .4654 02.0056810263184

So, we put in the following Tables (1-21) the time periods the satellites will be visible to each other. 
Table (1). EGYPTSAT_1 and TRMM are Visible During the Following Time Periods

\begin{tabular}{|c|c|c|c|c|c|c|c|c|c|c|c|c|}
\hline \multicolumn{3}{|c|}{ Date } & \multicolumn{3}{|c|}{ Time } & \multirow[b]{2}{*}{ To } & \multicolumn{3}{|c|}{ Date } & \multicolumn{3}{|c|}{ Time } \\
\hline$\underset{\nu}{\grave{\Xi}}$ & $\stackrel{\bar{E}}{E}$ & $\vec{E}$ & 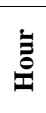 & 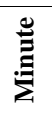 & 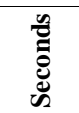 & & $\underset{\nabla}{\grave{J}}$ & ${ }^{\bar{E}}$ & है & $\stackrel{\Xi}{\Xi}$ & $\stackrel{\stackrel{\Xi}{\Xi}}{\stackrel{\Xi}{\Xi}}$ & 节 \\
\hline 2008 & 5 & 22 & 12 & 22 & 25.99 & & 2008 & 5 & 22 & 12 & 29 & 50.99 \\
\hline & & & 13 & 10 & 39.98 & & & & & 13 & 16 & 49.98 \\
\hline & & & 13 & 58 & 56.96 & t & & & & 14 & 3 & 41.96 \\
\hline & & & 14 & 47 & 26.95 & & & & & 14 & 50 & 24.94 \\
\hline & & & 22 & 43 & 18.79 & & & & & 22 & 46 & 30.79 \\
\hline & & & 23 & 30 & 05.78 & & & & & 23 & 34 & 54.77 \\
\hline 2008 & 5 & 23 & 0 & 16 & 56.77 & & 2008 & 5 & 23 & 0 & 23 & 15.76 \\
\hline & & & 1 & 3 & 58.74 & & & & & 1 & 11 & 25.74 \\
\hline & & & 1 & 50 & 59.73 & & & & & 1 & 59 & 35.73 \\
\hline & & & 2 & 38 & 07.71 & & & & & 2 & 47 & 37.71 \\
\hline & & & 3 & 25 & 15.70 & & & & & 3 & 35 & 40.70 \\
\hline & & & 4 & 12 & 30.68 & & & & & 4 & 23 & 36.68 \\
\hline & & & 4 & 59 & 45.67 & & & & & 5 & 11 & 31.66 \\
\hline & & & 5 & 47 & 06.65 & & & & & 5 & 59 & 20.65 \\
\hline & & & 6 & 34 & 28.64 & to & & & & 6 & 47 & 08.63 \\
\hline & & & 7 & 21 & 57.62 & & & & & 7 & 34 & 50.62 \\
\hline & & & 8 & 9 & 26.61 & & & & & 8 & 15 & 16.60 \\
\hline & & & 8 & 16 & 39.60 & & & & & 8 & 22 & 30.60 \\
\hline & & & 8 & 57 & 02.59 & & & & & 9 & 2 & 52.59 \\
\hline & & & 9 & 4 & 16.59 & & & & & 9 & 10 & 4.59 \\
\hline & & & 9 & 44 & 39.58 & & & & & 9 & 57 & 36.57 \\
\hline & & & 10 & 32 & 23.56 & & & & & 10 & 45 & 03.56 \\
\hline & & & 11 & 20 & 08.54 & & & & & 11 & 32 & 28.54 \\
\hline
\end{tabular}

Table 2. EGYPTSAT_1 and GOES_3 are Visible During the Following Time Periods

\begin{tabular}{|c|c|c|c|c|c|c|c|c|c|c|c|c|}
\hline \multicolumn{3}{|c|}{ Date } & \multicolumn{3}{|c|}{ Time } & \multirow[b]{2}{*}{ To } & \multicolumn{3}{|c|}{ Date } & \multicolumn{3}{|c|}{ Time } \\
\hline$\ddot{\Xi}$ & $\stackrel{\overrightarrow{0}}{\mathrm{E}}$ & $\vec{E}$ & $\stackrel{\Xi}{\Xi}$ & 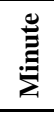 & 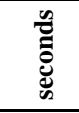 & & $\underset{\varpi}{\grave{\Xi}}$ & 音 & تี & $\stackrel{\Xi}{\Xi}$ & 总 & 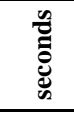 \\
\hline 2008 & 05 & 22 & 12 & 14 & 01.00 & & 2008 & 05 & 22 & 13 & 17 & 55.97 \\
\hline & & & 13 & 47 & 46.96 & & & & & 14 & 09 & 51.96 \\
\hline & & & 14 & 28 & 06.95 & & & & & 14 & 47 & 22.95 \\
\hline & & & 16 & 06 & 46.92 & & & & & 16 & 21 & 13.91 \\
\hline & & & 17 & 00 & 01.90 & & & & & 17 & 13 & 25.90 \\
\hline & & & 17 & 43 & 17.89 & to & & & & 17 & 56 & 41.88 \\
\hline & & & 18 & 35 & 44.87 & & & & & 18 & 49 & 30.87 \\
\hline & & & 21 & 41 & 22.81 & & & & & 22 & 44 & 05.79 \\
\hline & & & 23 & 04 & 17.78 & & 2008 & 05 & 23 & 00 & 08 & 51.76 \\
\hline
\end{tabular}


Table 2. cont..

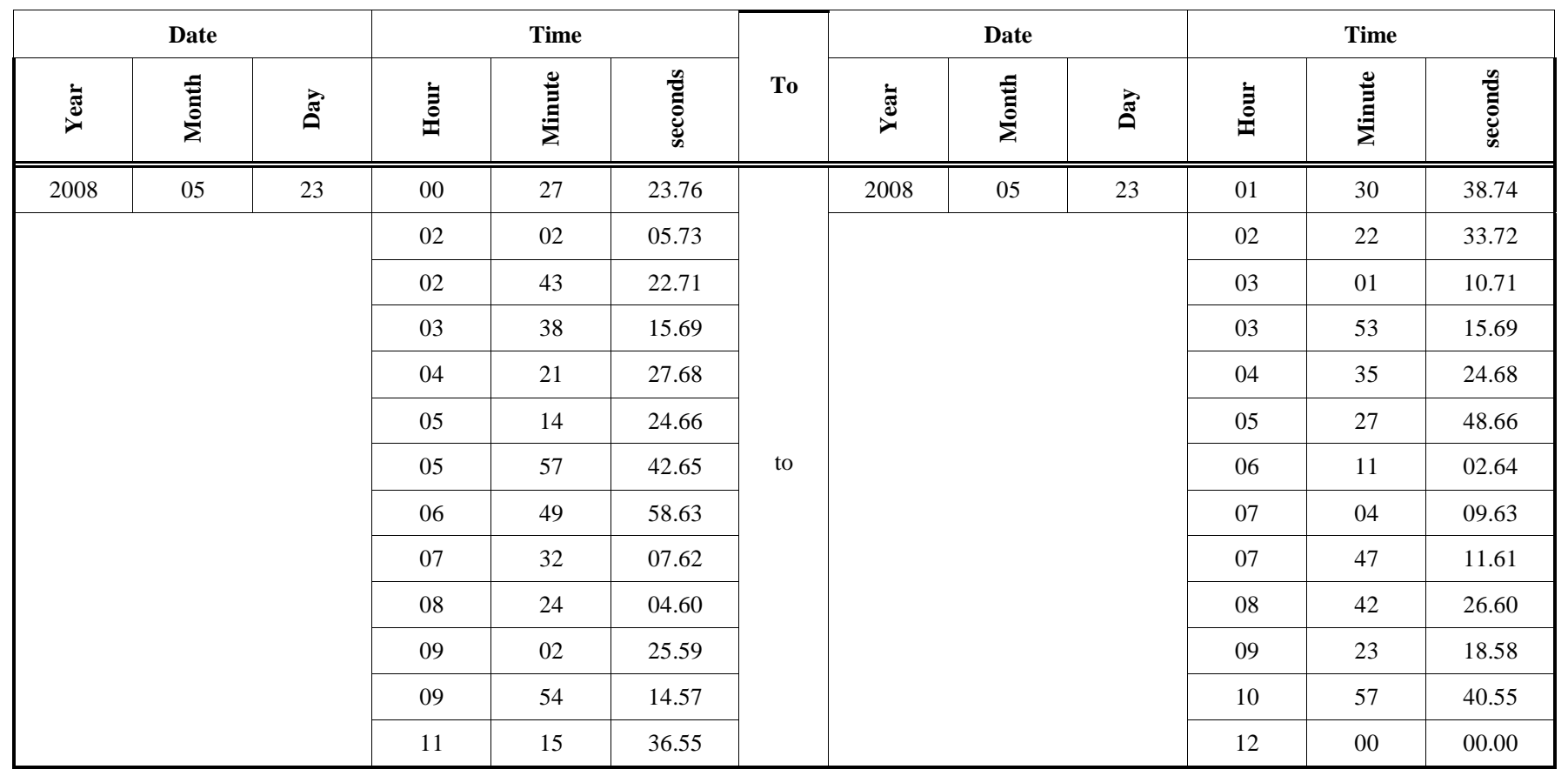

Table 3. GOES_3 and NAVSTAR_46 are Visible at the Time Periods

\begin{tabular}{|c|c|c|c|c|c|c|c|c|c|c|c|c|}
\hline \multicolumn{3}{|c|}{ Date } & \multicolumn{3}{|c|}{ Time } & \multirow[b]{2}{*}{ to } & \multicolumn{3}{|c|}{ Date } & \multicolumn{3}{|c|}{ Time } \\
\hline 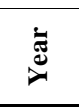 & $\stackrel{\bar{\Xi}}{\tilde{E}}$ & $\vec{\Xi}$ & $\stackrel{\Xi}{\Xi}$ & 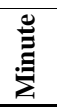 & 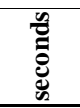 & & $\underset{\nu}{\grave{j}}$ & 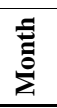 & $\vec{\theta}$ & $\stackrel{\Xi}{0}$ & 总 & $\begin{array}{l}n \\
\ddot{E} \\
\ddot{E} \\
\tilde{E}\end{array}$ \\
\hline
\end{tabular}

Table 4. GOES_3 and Hyp_1 are Visible at the Time Periods

\begin{tabular}{|c|c|c|c|c|c|c|c|c|c|c|c|c|}
\hline \multicolumn{3}{|c|}{ Date } & \multicolumn{3}{|c|}{ Time } & & \multicolumn{3}{|c|}{ Date } & \multicolumn{3}{|c|}{ Time } \\
\hline 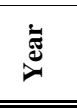 & 䏤 & $\vec{\Xi}$ & $\stackrel{\Xi}{\Xi}$ & $\stackrel{\stackrel{\Xi}{\Xi}}{\Xi}$ & 苛 & To & 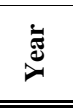 & $\stackrel{\underline{\Xi}}{\underline{\Xi}}$ & 己ి & $\stackrel{\Xi}{\Xi}$ & 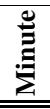 & 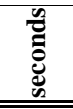 \\
\hline 2008 & 05 & 22 & 12 & 00 & 00.00 & & 2008 & 05 & 23 & 12 & 00 & 00.00 \\
\hline
\end{tabular}

Table 5. EGYPTSAT_1 and Hyp_1 are Visible During the Following Time Periods

\begin{tabular}{|c|c|c|c|c|c|c|c|c|c|c|c|c|}
\hline \multicolumn{3}{|c|}{ Date } & \multicolumn{3}{|c|}{ Time } & \multirow[b]{2}{*}{ To } & \multicolumn{3}{|c|}{ Date } & \multicolumn{3}{|c|}{ Time } \\
\hline$\stackrel{\vec{\Xi}}{\grave{J}}$ & 音 & $\overrightarrow{\mathrm{\theta}}$ & $\stackrel{\Xi}{\Xi}$ & $\stackrel{\Xi}{\Xi}$ & 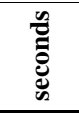 & & $\stackrel{\bar{\Xi}}{\grave{\nu}}$ & 竎 & $\vec{\theta}$ & $\stackrel{\Xi}{\Xi}$ & 总 & 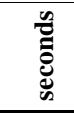 \\
\hline 2008 & 05 & 22 & 12 & 24 & 36.00 & \multirow{7}{*}{ to } & 2008 & 05 & 22 & 12 & 46 & 47.98 \\
\hline & & & 13 & 13 & 38.98 & & & & & 13 & 35 & 45.97 \\
\hline & & & 14 & 02 & 32.96 & & & & & 14 & 24 & 43.95 \\
\hline & & & 15 & 40 & 28.93 & & & & & 16 & 02 & 39.92 \\
\hline & & & 16 & 29 & 32.91 & & & & & 16 & 51 & 37.91 \\
\hline & & & 17 & 18 & 25.90 & & & & & 17 & 40 & 36.89 \\
\hline & & & 18 & 07 & 29.88 & & & & & 18 & 29 & 34.87 \\
\hline
\end{tabular}


Table 5. cont.

\begin{tabular}{|c|c|c|c|c|c|c|c|c|c|c|c|c|}
\hline \multicolumn{3}{|c|}{ Date } & \multicolumn{3}{|c|}{ Time } & \multirow[b]{2}{*}{ To } & \multicolumn{3}{|c|}{ Date } & \multicolumn{3}{|c|}{ Time } \\
\hline$\stackrel{\varpi}{\Xi}$ & $\stackrel{\bar{\Xi}}{\bar{E}}$ & $\vec{\Xi}$ & 苛 & $\underset{⿱ 乛}{\stackrel{\Xi}{\Xi}}$ & 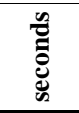 & & $\stackrel{\Xi}{\Xi}$ & $\stackrel{\bar{E}}{\bar{E}}$ & हैं & $\stackrel{\Xi}{\Xi}$ & $\stackrel{\Xi}{\Xi}$ & 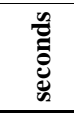 \\
\hline & & & 21 & 23 & 23.82 & & & & & 21 & 45 & 26.81 \\
\hline & & & 22 & 12 & 17.80 & & & & & 22 & 34 & 25.79 \\
\hline & & & 23 & 01 & 20.78 & & & & & 23 & 23 & 23.78 \\
\hline & & & 23 & 50 & 14.77 & & 2008 & 05 & 23 & 00 & 12 & 22.76 \\
\hline 2008 & 05 & 23 & 00 & 39 & 17.75 & & 2008 & 05 & 23 & 01 & 01 & 19.75 \\
\hline & & & 01 & 28 & 11.74 & & & & & 01 & 50 & 18.73 \\
\hline & & & 02 & 17 & 14.72 & & & & & 02 & 39 & 16.71 \\
\hline & & & 03 & 06 & 08.71 & & & & & 03 & 28 & 15.70 \\
\hline & & & 03 & 55 & 11.69 & & & & & 04 & 17 & 13.68 \\
\hline & & & 04 & 44 & 05.67 & & & & & 05 & 06 & 12.67 \\
\hline & & & 05 & 33 & 08.66 & & & & & 05 & 55 & 09.65 \\
\hline & & & 06 & 22 & 02.64 & to & & & & 06 & 44 & 08.63 \\
\hline & & & 07 & 11 & 05.63 & & & & & 07 & 33 & 06.62 \\
\hline & & & 08 & 00 & 00.61 & & & & & 08 & 22 & 05.60 \\
\hline & & & 08 & 49 & 03.59 & & & & & 09 & 11 & 03.59 \\
\hline & & & 09 & 37 & 57.58 & & & & & 10 & 00 & 02.57 \\
\hline & & & 10 & 27 & 00.56 & & & & & 10 & 49 & 00.55 \\
\hline & & & 11 & 15 & 54.55 & & & & & 11 & 37 & 59.54 \\
\hline
\end{tabular}

Table 6. EGYPTSAT_1 and NOAA_3 are Visible During the Following Time Periods

\begin{tabular}{|c|c|c|c|c|c|c|c|c|c|c|c|c|}
\hline \multicolumn{3}{|c|}{ Date } & \multicolumn{3}{|c|}{ Time } & \multirow[b]{2}{*}{ To } & \multicolumn{3}{|c|}{ Date } & \multicolumn{3}{|c|}{ Time } \\
\hline 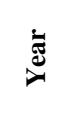 & 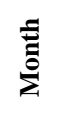 & $\vec{\theta}$ & $\stackrel{\Xi}{\Xi}$ & $\stackrel{\Xi}{\Xi}$ & 总 & & 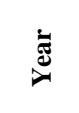 & 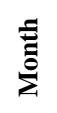 & ڤ્తి & $\dot{\Xi}$ & $\stackrel{\Xi}{\Xi}$ & 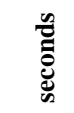 \\
\hline 2008 & 05 & 22 & 14 & 28 & 15.95 & \multirow{4}{*}{ To } & 2008 & 05 & 22 & 14 & 45 & 48.95 \\
\hline & & & 15 & 05 & 04.94 & & & & & 16 & 15 & 10.92 \\
\hline & & & 16 & 23 & 09.91 & & & & & 17 & 35 & 35.89 \\
\hline & & & 17 & 48 & 02.89 & & & & & 18 & 12 & 30.88 \\
\hline 2008 & 05 & 23 & 01 & 02 & 00.75 & \multirow{5}{*}{ To } & 2008 & 05 & 23 & 01 & 30 & 10.74 \\
\hline & & & 01 & 37 & 54.73 & & & & & 02 & 52 & 28.71 \\
\hline & & & 03 & 00 & 10.71 & & & & & 04 & 09 & 27.68 \\
\hline & & & 04 & 32 & 23.68 & & & & & 04 & 45 & 18.67 \\
\hline & & & 11 & 36 & 21.54 & & & & & 12 & 00 & 00.00 \\
\hline
\end{tabular}

Table 7. EGYPTSAT_1 and NAVSTAR_46 are Visible During the Following Time Periods

\begin{tabular}{|c|c|c|c|c|c|c|c|c|c|c|c|c|}
\hline \multicolumn{3}{|c|}{ Date } & \multicolumn{3}{|c|}{ Time } & \multirow[b]{2}{*}{ To } & \multicolumn{3}{|c|}{ Date } & \multicolumn{3}{|c|}{ Time } \\
\hline$\stackrel{\vec{\varpi}}{\grave{\nu}}$ & $\bar{\Xi}$ & $\vec{\theta}$ & $\stackrel{\Xi}{\Xi}$ & 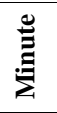 & 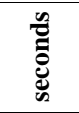 & & 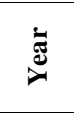 & 离 & $\vec{\Xi}$ & $\stackrel{\Xi}{\Xi}$ & $\stackrel{\Xi}{\Xi}$ & 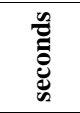 \\
\hline 2008 & 05 & 22 & 12 & 36 & 44.99 & \multirow{3}{*}{ To } & 2008 & 05 & 22 & 13 & 00 & 36.98 \\
\hline & & & 13 & 24 & 12.97 & & & & & 13 & 43 & 46.97 \\
\hline & & & 14 & 31 & 20.95 & & & & & 14 & 46 & 41.95 \\
\hline
\end{tabular}


Table 7. cont...

\begin{tabular}{|c|c|c|c|c|c|c|c|c|c|c|c|c|}
\hline \multicolumn{3}{|c|}{ Date } & \multicolumn{3}{|c|}{ Time } & \multirow[b]{2}{*}{ To } & \multicolumn{3}{|c|}{ Date } & \multicolumn{3}{|c|}{ Time } \\
\hline$\underset{ઇ}{\Xi}$ & 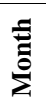 & $\overrightarrow{0}$ & $\stackrel{\Xi}{\Xi}$ & $\stackrel{\Xi}{\Xi}$ & 氖 & & 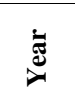 & 忢 & $\vec{\theta}$ & $\stackrel{\Xi}{\Xi}$ & $\stackrel{\circlearrowright}{\Xi}$ & 电 \\
\hline & & & 15 & 17 & 10.94 & \multirow{23}{*}{ To } & & & & 15 & 32 & 15.93 \\
\hline & & & 16 & 19 & 53.92 & & & & & 16 & 38 & 47.91 \\
\hline & & & 17 & 03 & 22.90 & & & & & 17 & 26 & 13.89 \\
\hline & & & 18 & 16 & 31.88 & & & & & 18 & 42 & 08.87 \\
\hline & & & 19 & 3 & 34.86 & & & & & 19 & 25 & 5.86 \\
\hline & & & 20 & 12 & 43.84 & & & & & 20 & 28 & 17.83 \\
\hline & & & 20 & 58 & 39.82 & & & & & 21 & 13 & 44.82 \\
\hline & & & 22 & 00 & 55.80 & & & & & 22 & 18 & 07.80 \\
\hline & & & 22 & 44 & 51.79 & & & & & 23 & 05 & 18.78 \\
\hline & & & 23 & 54 & 31.77 & & 2008 & 05 & 23 & 00 & 22 & 29.76 \\
\hline 2008 & 05 & \multirow[t]{13}{*}{23} & 00 & 40 & 11.75 & & 2008 & 05 & 23 & 01 & 04 & 57.74 \\
\hline & & & 01 & 53 & 01.73 & & & & & 02 & 09 & 41.72 \\
\hline & & & 02 & 39 & 27.71 & & & & & 02 & 54 & 37.71 \\
\hline & & & 03 & 42 & 03.69 & & & & & 03 & 58 & 18.69 \\
\hline & & & 04 & 26 & 29.68 & & & & & 04 & 45 & 10.67 \\
\hline & & & 05 & 34 & 23.66 & & & & & 06 & 02 & 40.65 \\
\hline & & & 06 & 18 & 35.64 & & & & & 06 & 45 & 39.63 \\
\hline & & & 07 & 34 & 06.62 & & & & & 07 & 51 & 27.61 \\
\hline & & & 08 & 20 & 37.60 & & & & & 08 & 36 & 16.60 \\
\hline & & & 09 & 23 & 24.58 & & & & & 09 & 38 & 52.58 \\
\hline & & & 10 & 08 & 08.57 & & & & & 10 & 25 & 27.56 \\
\hline & & & 11 & 13 & 33.55 & & & & & 11 & 40 & 00.54 \\
\hline & & & 11 & 56 & 16.53 & & & & & 12 & 00 & 00.00 \\
\hline
\end{tabular}

Table 8. EGYPTSAT_1 and Parabola are Visible at the Time Periods

\begin{tabular}{|c|c|c|c|c|c|c|c|c|c|c|c|c|}
\hline \multicolumn{3}{|c|}{ Date } & \multicolumn{3}{|c|}{ Time } & \multirow[b]{2}{*}{ To } & \multicolumn{3}{|c|}{ Date } & \multicolumn{3}{|c|}{ Time } \\
\hline$\underset{\succsim}{ઇ}$ & 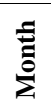 & పే & $\ddot{\Xi}$ & $\stackrel{\stackrel{\Xi}{\Xi}}{\Xi}$ & 氖 & & $\underset{\varpi}{\rightleftarrows}$ & 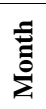 & 苛 & $\stackrel{\Xi}{\Xi}$ & $\underset{\Xi}{\stackrel{\Xi}{\Xi}}$ & 氖 \\
\hline 2008 & 05 & 22 & 12 & 00 & 00.00 & \multirow{12}{*}{ to } & 2008 & 05 & 22 & 12 & 01 & 50.00 \\
\hline & & & 12 & 29 & 50.99 & & & & & 12 & 50 & 53.98 \\
\hline & & & 13 & 18 & 51.97 & & & & & 13 & 39 & 47.97 \\
\hline & & & 14 & 07 & 48.96 & & & & & 14 & 28 & 51.95 \\
\hline & & & 14 & 56 & 49.94 & & & & & 15 & 17 & 45.94 \\
\hline & & & 15 & 45 & 46.93 & & & & & 16 & 06 & 49.92 \\
\hline & & & 16 & 34 & 47.91 & & & & & 16 & 55 & 43.90 \\
\hline & & & 17 & 23 & 44.89 & & & & & 17 & 44 & 47.89 \\
\hline & & & 18 & 12 & 45.88 & & & & & 18 & 33 & 41.87 \\
\hline & & & 19 & 01 & 42.86 & & & & & 19 & 22 & 45.86 \\
\hline & & & 19 & 50 & 44.85 & & & & & 20 & 11 & 39.84 \\
\hline & & & 20 & 39 & 40.83 & & & & & 21 & 00 & 43.82 \\
\hline
\end{tabular}


Table 8. cont...

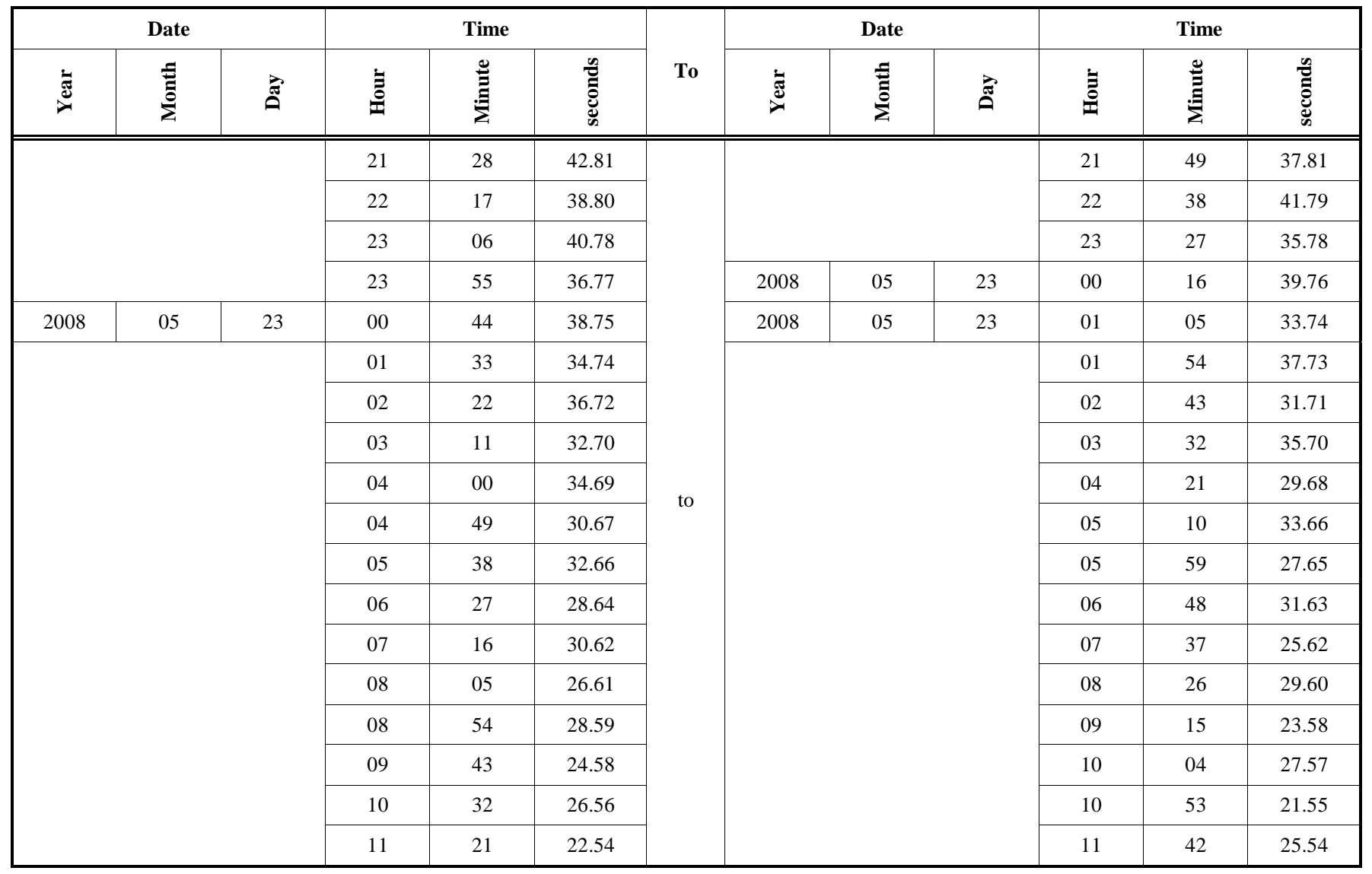

Table 9. GOES_3 and Parabola are Visible at the Time Periods

\begin{tabular}{|c|c|c|c|c|c|c|c|c|c|c|c|c|}
\hline \multicolumn{3}{|c|}{ Date } & \multicolumn{3}{|c|}{ Time } & \multirow{3}{*}{ To } & \multicolumn{3}{|c|}{ Date } & \multicolumn{3}{|c|}{ Time } \\
\hline$\stackrel{\vec{\Xi}}{\grave{\nu}}$ & 莣 & $\vec{E}$ & $\stackrel{\Xi}{\Xi}$ & $\stackrel{\Xi}{\Xi}$ & 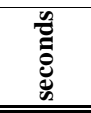 & & 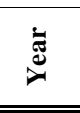 & 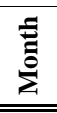 & $\vec{\Xi}$ & $\stackrel{\grave{\Xi}}{\Xi}$ & 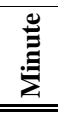 & 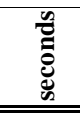 \\
\hline 2008 & 05 & 22 & 12 & 00 & 00.00 & & 2008 & 05 & 23 & 12 & 00 & 00.00 \\
\hline
\end{tabular}

Table 10. TRMM and GOES_3 are Visible During the Following Time Periods

\begin{tabular}{|c|c|c|c|c|c|c|c|c|c|c|c|c|}
\hline \multicolumn{3}{|c|}{ Date } & \multicolumn{3}{|c|}{ Time } & \multirow[b]{2}{*}{ To } & \multicolumn{3}{|c|}{ Date } & \multicolumn{3}{|c|}{ Time } \\
\hline$\dot{\bar{\Xi}}$ & $\stackrel{\bar{E}}{\sum}$ & $\overrightarrow{\mathrm{\theta}}$ & 志 & $\stackrel{\Xi}{\stackrel{\Xi}{\Xi}}$ & 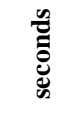 & & $\underset{\nabla}{\nexists ~}$ & 莫 & $\vec{E}$ & 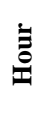 & $\stackrel{\Xi}{:}$ & 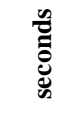 \\
\hline 2008 & 05 & 22 & 12 & 09 & 00.00 & \multirow{11}{*}{ to } & 2008 & 05 & 22 & 12 & 21 & 07.99 \\
\hline & & & 13 & 04 & 05.98 & & & & & 13 & 16 & 32.98 \\
\hline & & & 13 & 48 & 23.96 & & & & & 14 & 00 & 55.96 \\
\hline & & & 14 & 43 & 53.95 & & & & & 14 & 56 & 16.94 \\
\hline & & & 15 & 28 & 19.93 & & & & & 15 & 40 & 30.93 \\
\hline & & & 16 & 23 & 29.91 & & & & & 16 & 35 & 12.91 \\
\hline & & & 17 & 07 & 57.90 & & & & & 17 & 19 & 24.90 \\
\hline & & & 18 & 02 & 24.88 & & & & & 18 & 13 & 28.88 \\
\hline & & & 18 & 46 & 49.87 & & & & & 18 & 57 & 43.86 \\
\hline & & & 19 & 40 & 45.85 & & & & & 19 & 51 & 31.85 \\
\hline & & & 20 & 25 & 03.84 & & & & & 20 & 35 & 52.83 \\
\hline
\end{tabular}


Table 10. cont...

\begin{tabular}{|c|c|c|c|c|c|c|c|c|c|c|c|c|}
\hline \multicolumn{3}{|c|}{ Date } & \multicolumn{3}{|c|}{ Time } & \multirow[b]{2}{*}{ To } & \multicolumn{3}{|c|}{ Date } & \multicolumn{3}{|c|}{ Time } \\
\hline 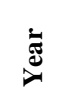 & 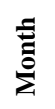 & $\vec{\Xi}$ & $\stackrel{\Xi}{\Xi}$ & $\stackrel{\stackrel{\Xi}{\Xi}}{:}$ & 晜 & & 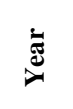 & 䒿 & $\ddot{\Xi}$ & $\stackrel{\Xi}{\ddot{\theta}}$ & $\stackrel{\stackrel{\Xi}{\Xi}}{\stackrel{\Xi}{\Xi}}$ & 电 \\
\hline \multirow[b]{5}{*}{2008} & & \multirow[b]{5}{*}{23} & 21 & 18 & 54.82 & & & & & 21 & 29 & 53.81 \\
\hline & & & 22 & 03 & 06.80 & & & & & 22 & 14 & 20.80 \\
\hline & & & 22 & 57 & 20.79 & & & & & 23 & 08 & 58.78 \\
\hline & & & 23 & 41 & 31.77 & & & & & 23 & 53 & 28.77 \\
\hline & 05 & & 00 & 36 & 25.75 & \multirow{14}{*}{ to } & 2008 & 05 & 23 & 00 & 48 & 46.75 \\
\hline & & & 01 & 20 & 42.74 & & & & & 01 & 33 & 11.74 \\
\hline & & & 02 & 16 & 08.72 & & & & & 02 & 28 & 38.72 \\
\hline & & & 03 & 00 & 34.71 & & & & & 03 & 12 & 53.70 \\
\hline & & & 03 & 55 & 51.69 & & & & & 04 & 07 & 48.69 \\
\hline & & & 04 & 40 & 21.67 & & & & & 04 & 51 & 58.67 \\
\hline & & & 05 & 34 & 58.66 & & & & & 05 & 46 & 11.65 \\
\hline & & & 06 & 19 & 24.64 & & & & & 06 & 30 & 23.64 \\
\hline & & & 07 & 13 & 25.62 & & & & & 07 & 24 & 14.62 \\
\hline & & & 07 & 57 & 46.61 & & & & & 08 & 08 & 32.61 \\
\hline & & & 08 & 51 & 34.59 & & & & & 09 & 02 & 29.59 \\
\hline & & & 09 & 35 & 49.58 & & & & & 09 & 46 & 53.57 \\
\hline & & & 10 & 29 & 54.56 & & & & & 10 & 41 & 21.56 \\
\hline & & & 11 & 14 & 05.55 & & & & & 11 & 25 & 50.54 \\
\hline
\end{tabular}

Table 11. NAVSTAR_46 and Hyp_1 are Visible at the Time Periods

\begin{tabular}{|c|c|c|c|c|c|c|c|c|c|c|c|c|}
\hline \multicolumn{3}{|c|}{ Date } & \multicolumn{3}{|c|}{ Time } & \multirow[b]{2}{*}{ To } & \multicolumn{3}{|c|}{ Date } & \multicolumn{3}{|c|}{ Time } \\
\hline 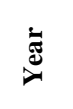 & $\stackrel{\Xi}{\underline{\Xi}}$ & $\vec{\Xi}$ & $\stackrel{\Xi}{\Xi}$ & $\stackrel{\stackrel{\Xi}{\Xi}}{:}$ & 葛 & & $\stackrel{\Xi}{\varpi}$ & $\stackrel{\Xi}{\Xi}$ & ڤ્ષ & $\stackrel{\Xi}{\Xi}$ & $\stackrel{\stackrel{\Xi}{\Xi}}{\stackrel{\Xi}{\Xi}}$ & 氖 \\
\hline 2008 & 05 & 22 & 12 & 00 & 00.00 & to & 2008 & 05 & 23 & 12 & 00 & 00.00 \\
\hline
\end{tabular}

Table 12. NOAA_3 and NAVSTAR_46 are Visible During the Following Time Periods

\begin{tabular}{|c|c|c|c|c|c|c|c|c|c|c|c|c|}
\hline \multicolumn{3}{|c|}{ Date } & \multicolumn{3}{|c|}{ Time } & \multirow[b]{2}{*}{ To } & \multicolumn{3}{|c|}{ Date } & \multicolumn{3}{|c|}{ Time } \\
\hline 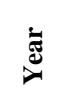 & 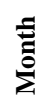 & $\vec{\Xi}$ & $\stackrel{\Xi}{\Xi}$ & $\stackrel{\stackrel{\Xi}{\Xi}}{\Xi}$ & 峞 & & $\stackrel{ઇ}{\beth}$ & $\stackrel{\Xi}{\underline{\Xi}}$ & 己ે & $\stackrel{\Xi}{\Xi}$ & $\stackrel{\stackrel{\Xi}{\Xi}}{\Xi}$ & 节 \\
\hline 2008 & 05 & 22 & 12 & 00 & 00.00 & \multirow{9}{*}{ to } & 2008 & 05 & 22 & 12 & 29 & 07.99 \\
\hline & & & 12 & 52 & 10.98 & & & & & 13 & 18 & 05.97 \\
\hline & & & 14 & 04 & 13.96 & & & & & 14 & 33 & 41.95 \\
\hline & & & 14 & 49 & 07.94 & & & & & 15 & 31 & 47.93 \\
\hline & & & 16 & 2 & 38.92 & & & & & 18 & 05 & 31.88 \\
\hline & & & 18 & 50 & 24.87 & & & & & 19 & 15 & 30.86 \\
\hline & & & 19 & 42 & 37.85 & & & & & 20 & 08 & 04.84 \\
\hline & & & 20 & 52 & 22.83 & & & & & 22 & 55 & 21.79 \\
\hline & & & 23 & 27 & 07.78 & & 2008 & 05 & 23 & 00 & 08 & 10.76 \\
\hline
\end{tabular}


Table 12. cont...

\begin{tabular}{|c|c|c|c|c|c|c|c|c|c|c|c|c|}
\hline \multicolumn{3}{|c|}{ Date } & \multicolumn{3}{|c|}{ Time } & \multirow[b]{2}{*}{ To } & \multicolumn{3}{|c|}{ Date } & \multicolumn{3}{|c|}{ Time } \\
\hline$\underset{\nabla}{\ddot{\Xi}}$ & $\stackrel{\bar{E}}{\bar{E}}$ & है & $\stackrel{\Xi}{0}$ & 莽 & 节 & & $\stackrel{\bar{\Xi}}{ \pm}$ & 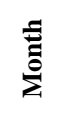 & $\stackrel{\vec{\theta}}{\theta}$ & 言 & $\stackrel{\Xi}{\Xi}$ & 号 \\
\hline 2008 & 05 & 23 & 00 & 24 & 29.76 & & 2008 & 05 & 23 & 00 & 53 & 40.75 \\
\hline & & & & 39 & 46.73 & & & & & 02 & 05 & 44.72 \\
\hline & & & & 28 & 29.72 & & & & & 03 & 01 & 00.71 \\
\hline & & & & 39 & 46.69 & to & & & & 05 & 41 & 30.65 \\
\hline & & & & 24 & 50.64 & & & & & 06 & 51 & 32.63 \\
\hline & & & & 18 & 10.62 & & & & & 07 & 42 & 53.62 \\
\hline & & & & 28 & 18.60 & & & & & 12 & 00 & 00.00 \\
\hline
\end{tabular}

Table 13. NAVSTAR_46 and Parabola are Visible at the Time Periods

\begin{tabular}{|c|c|c|c|c|c|c|c|c|c|c|c|c|}
\hline \multicolumn{3}{|c|}{ Date } & \multicolumn{3}{|c|}{ Time } & \multirow[b]{2}{*}{ To } & \multicolumn{3}{|c|}{ Date } & \multicolumn{3}{|c|}{ Time } \\
\hline$\stackrel{\dot{\varpi}}{\grave{\nu}}$ & 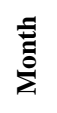 & $\vec{E}$ & $\stackrel{\Xi}{\Xi}$ & $\stackrel{\Xi}{\Xi}$ & 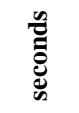 & & 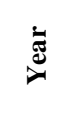 & 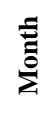 & $\vec{\Xi}$ & 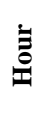 & 萻 & 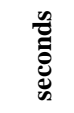 \\
\hline 2008 & 05 & 22 & 12 & 00 & 00.00 & to & 2008 & 05 & 23 & 12 & 00 & 00.00 \\
\hline
\end{tabular}

Table 14. Hyp_1 and Parabola are Visible During the Following Time Periods

\begin{tabular}{|c|c|c|c|c|c|c|c|c|c|c|c|c|}
\hline \multicolumn{3}{|c|}{ Date } & \multicolumn{3}{|c|}{ Time } & \multirow[b]{2}{*}{ To } & \multicolumn{3}{|c|}{ Date } & \multicolumn{3}{|c|}{ Time } \\
\hline$\stackrel{\bar{\Xi}}{\doteq}$ & $\overline{\bar{E}}$ & $\vec{\Xi}$ & $\stackrel{\Xi}{\Xi}$ & $\stackrel{\Xi}{\Xi}$ & 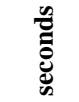 & & 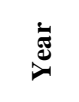 & $\stackrel{\bar{\Xi}}{\tilde{z}}$ & $\vec{\Xi}$ & 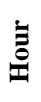 & 莣 & $\begin{array}{l}\tilde{E} \\
\ddot{E} \\
\mathbb{E} \\
\mathscr{E}\end{array}$ \\
\hline 2008 & 05 & 22 & 12 & 00 & 00.00 & to & 2008 & 05 & 23 & 12 & 00 & 00.00 \\
\hline
\end{tabular}

Table 15. TRMM and NAVSTAR_46 are Visible During the Following Time Periods

\begin{tabular}{|c|c|c|c|c|c|c|c|c|c|c|c|c|}
\hline \multicolumn{3}{|c|}{ Date } & \multicolumn{3}{|c|}{ Time } & \multirow[b]{2}{*}{ To } & \multicolumn{3}{|c|}{ Date } & \multicolumn{3}{|c|}{ Time } \\
\hline$\dot{\vec{\Xi}}$ & 总 & $\vec{E}$ & $\stackrel{\Xi}{\Xi}$ & $\stackrel{\Xi}{\Xi}$ & 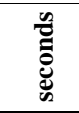 & & $\underset{\bar{~}}{\grave{\Xi}}$ & 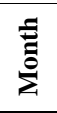 & $\vec{\Xi}$ & $\stackrel{\Xi}{\Xi}$ & 总 & 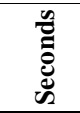 \\
\hline \multirow[t]{12}{*}{2008} & 05 & \multirow[t]{12}{*}{22} & 12 & 00 & 00.00 & \multirow{12}{*}{ to } & 2008 & 05 & 22 & 13 & 26 & 57.97 \\
\hline & & & 14 & 09 & 13.96 & & & & & 14 & 22 & 11.95 \\
\hline & & & 14 & 48 & 40.95 & & & & & 14 & 59 & 40.94 \\
\hline & & & 15 & 43 & 21.93 & & & & & 15 & 53 & 54.92 \\
\hline & & & 16 & 21 & 40.91 & & & & & 16 & 33 & 28.91 \\
\hline & & & 17 & 16 & 17.90 & & & & & 18 & 51 & 40.87 \\
\hline & & & 19 & 31 & 35.85 & & & & & 19 & 49 & 13.85 \\
\hline & & & 20 & 10 & 03.84 & & & & & 20 & 23 & 28.84 \\
\hline & & & 21 & 06 & 54.82 & & & & & 21 & 17 & 13.82 \\
\hline & & & 21 & 46 & 20.81 & & & & & 21 & 56 & 49.81 \\
\hline & & & 22 & 40 & 08.79 & & & & & 22 & 55 & 04.79 \\
\hline & & & 23 & 12 & 25.78 & & & & & 23 & 32 & 57.77 \\
\hline 2008 & 05 & 23 & 00 & 10 & 56.76 & & 2008 & 05 & 23 & 01 & 47 & 17.73 \\
\hline & & & 02 & 30 & 15.72 & to & & & & 02 & 41 & 39.71 \\
\hline & & & 03 & 09 & 54.70 & & & & & 03 & 20 & 16.70 \\
\hline
\end{tabular}


Table 15. cont...

\begin{tabular}{|c|c|c|c|c|c|c|c|c|c|c|c|c|}
\hline \multicolumn{3}{|c|}{ Date } & \multicolumn{3}{|c|}{ Time } & \multirow[b]{2}{*}{ To } & \multicolumn{3}{|c|}{ Date } & \multicolumn{3}{|c|}{ Time } \\
\hline$\stackrel{\Xi}{\varpi}$ & 莽 & $\vec{\nabla}$ & $\ddot{\Xi}$ & $\stackrel{\stackrel{\Xi}{\Xi}}{\sum^{3}}$ & $\underset{\tilde{Z}}{\tilde{E}}$ & & $\underset{\partial}{\beth}$ & 馬 & $\overrightarrow{\mathrm{D}}$ & $\stackrel{\Xi}{\Xi}$ & $\stackrel{\stackrel{\Xi}{\Xi}}{\Xi}$ & $\underset{\mathscr{Z}}{\mathscr{Z}}$ \\
\hline & & & 04 & 03 & 57.69 & & & & & 04 & 15 & 20.68 \\
\hline & & & 04 & 40 & 51.67 & & & & & 04 & 54 & 36.67 \\
\hline & & & 05 & 36 & 33.66 & & & & & 07 & 11 & 25.63 \\
\hline & & & 07 & 53 & 13.61 & & & & & 08 & 07 & 08.61 \\
\hline & & & 08 & 32 & 30.60 & & & & & 08 & 44 & 01.60 \\
\hline & & & 09 & 27 & 34.58 & & & & & 09 & 37 & 53.58 \\
\hline & & & 10 & 06 & 27.57 & & & & & 10 & 17 & 37.56 \\
\hline & & & 11 & 00 & 35.55 & & & & & 11 & 58 & 44.53 \\
\hline
\end{tabular}

Table 16. TRMM and NOAA_3 are Visible During the Following Time Periods

\begin{tabular}{|c|c|c|c|c|c|c|c|c|c|c|c|c|}
\hline \multicolumn{3}{|c|}{ Date } & \multicolumn{3}{|c|}{ Time } & \multirow[b]{2}{*}{ To } & \multicolumn{3}{|c|}{ Date } & \multicolumn{3}{|c|}{ Time } \\
\hline 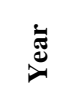 & 馬 & $\vec{\Xi}$ & $\stackrel{\Xi}{\Xi}$ & $\underset{\Xi}{\stackrel{\Xi}{\Xi}}$ & 芯 & & $\underset{\varpi}{\varpi}$ & 䒿 & $\vec{\Xi}$ & $\stackrel{\Xi}{\Xi}$ & $\stackrel{\stackrel{\Xi}{\Xi}}{\Xi}$ & 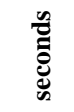 \\
\hline 2008 & 05 & 22 & 12 & 00 & 00.00 & \multirow{11}{*}{ to } & 2008 & 05 & 22 & 12 & 02 & 45.00 \\
\hline \multirow[b]{11}{*}{2008} & \multirow[b]{11}{*}{05} & & 12 & 04 & 13.00 & & & & & 12 & 12 & 32.00 \\
\hline & & & 12 & 47 & 40.98 & & & & & 13 & 01 & 50.98 \\
\hline & & & 13 & 43 & 07.97 & & & & & 13 & 49 & 28.96 \\
\hline & & & 17 & 07 & 14.90 & & & & & 17 & 18 & 51.90 \\
\hline & & & 17 & 55 & 59.88 & & & & & 18 & 12 & 55.88 \\
\hline & & & 18 & 46 & 07.87 & & & & & 18 & 52 & 59.87 \\
\hline & & & 18 & 58 & 23.86 & & & & & 19 & 05 & 15.86 \\
\hline & & & 19 & 37 & 59.85 & & & & & 19 & 55 & 47.85 \\
\hline & & & 20 & 31 & 34.83 & & & & & 20 & 44 & 55.83 \\
\hline & & & 21 & 27 & 32.82 & & & & & 21 & 32 & 05.81 \\
\hline & & \multirow[t]{15}{*}{23} & 00 & 03 & 53.76 & \multirow{15}{*}{ to } & 2008 & 05 & 23 & 00 & 06 & 00.76 \\
\hline & & & 00 & 50 & 15.75 & & & & & 01 & 02 & 49.75 \\
\hline & & & 01 & 39 & 12.73 & & & & & 01 & 56 & 38.73 \\
\hline & & & 02 & 29 & 33.72 & & & & & 02 & 36 & 24.71 \\
\hline & & & 02 & 41 & 53.71 & & & & & 02 & 48 & 43.71 \\
\hline & & & 03 & 21 & 40.70 & & & & & 03 & 39 & 02.69 \\
\hline & & & 04 & 15 & 30.68 & & & & & 04 & 27 & 58.68 \\
\hline & & & 05 & 12 & 36.66 & & & & & 05 & 14 & 03.66 \\
\hline & & & 07 & 46 & 03.61 & & & & & 07 & 50 & 52.61 \\
\hline & & & 08 & 33 & 19.60 & & & & & 08 & 46 & 45.59 \\
\hline & & & 09 & 22 & 27.58 & & & & & 09 & 40 & 19.58 \\
\hline & & & 10 & 13 & 01.57 & & & & & 10 & 19 & 54.56 \\
\hline & & & 10 & 25 & 17.56 & & & & & 10 & 32 & 9.56 \\
\hline & & & 11 & 05 & 23.55 & & & & & 11 & 22 & 15.54 \\
\hline & & & 11 & 59 & 28.53 & & & & & 12 & 00 & 00.00 \\
\hline
\end{tabular}


Table 17. TRMM and Hyp_1 are Visible During the Following Time Periods

\begin{tabular}{|c|c|c|c|c|c|c|c|c|c|c|c|c|}
\hline \multicolumn{3}{|c|}{ Date } & \multicolumn{3}{|c|}{ Time } & \multirow[b]{2}{*}{ To } & \multicolumn{3}{|c|}{ Date } & \multicolumn{3}{|c|}{ Time } \\
\hline$\underset{\nabla}{\vec{\Xi}}$ & 总 & $\vec{E}$ & $\stackrel{\Xi}{\Xi}$ & 晜 & 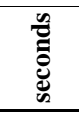 & & $\stackrel{\grave{\Xi}}{\bar{\Xi}}$ & 离 & $\stackrel{\theta}{\theta}$ & $\stackrel{\Xi}{0}$ & $\stackrel{\Xi}{\Xi}$ & 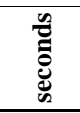 \\
\hline \multirow[t]{16}{*}{2008} & 05 & 22 & 12 & 10 & 49.00 & \multirow{16}{*}{ to } & 2008 & 05 & 22 & 12 & 20 & 58.99 \\
\hline & & & 12 & 57 & 3.98 & & & & & 13 & 7 & 14.98 \\
\hline & & & 13 & 43 & 22.97 & & & & & 13 & 53 & 32.96 \\
\hline & & & 14 & 29 & 37.95 & & & & & 14 & 39 & 48.95 \\
\hline & & & 15 & 15 & 56.94 & & & & & 15 & 26 & 06.93 \\
\hline & & & 16 & 02 & 11.92 & & & & & 16 & 12 & 22.92 \\
\hline & & & 16 & 48 & 30.91 & & & & & 16 & 58 & 41.90 \\
\hline & & & 17 & 34 & 45.89 & & & & & 17 & 44 & 56.89 \\
\hline & & & 18 & 21 & 04.88 & & & & & 18 & 31 & 15.87 \\
\hline & & & 19 & 07 & 19.86 & & & & & 19 & 17 & 30.86 \\
\hline & & & 19 & 53 & 38.85 & & & & & 20 & 3 & 48.84 \\
\hline & & & 20 & 39 & 53.83 & & & & & 20 & 50 & 04.83 \\
\hline & & & 21 & 26 & 12.82 & & & & & 21 & 36 & 22.81 \\
\hline & & & 22 & 12 & 27.80 & & & & & 22 & 22 & 38.80 \\
\hline & & & 22 & 58 & 46.79 & & & & & 23 & 8 & 56.78 \\
\hline & & & 23 & 45 & 01.77 & & & & & 23 & 55 & 12.77 \\
\hline \multirow[t]{15}{*}{2008} & 05 & 23 & 00 & 31 & 20.76 & \multirow{15}{*}{ to } & 2008 & 05 & 23 & 00 & 41 & 30.75 \\
\hline & & & 01 & 17 & 35.74 & & & & & 01 & 27 & 46.74 \\
\hline & & & 02 & 03 & 54.73 & & & & & 02 & 14 & 04.72 \\
\hline & & & 02 & 50 & 09.71 & & & & & 03 & 00 & 20.71 \\
\hline & & & 03 & 36 & 28.70 & & & & & 03 & 46 & 38.69 \\
\hline & & & 04 & 22 & 43.68 & & & & & 04 & 32 & 54.68 \\
\hline & & & 05 & 09 & 01.67 & & & & & 05 & 19 & 12.66 \\
\hline & & & 05 & 55 & 17.65 & & & & & 06 & 05 & 27.65 \\
\hline & & & 06 & 41 & 35.63 & & & & & 06 & 51 & 46.63 \\
\hline & & & 07 & 27 & 51.62 & & & & & 07 & 38 & 01.62 \\
\hline & & & 08 & 14 & 09.60 & & & & & 08 & 24 & 19.60 \\
\hline & & & 09 & 00 & 24.59 & & & & & 09 & 10 & 35.59 \\
\hline & & & 09 & 46 & 43.57 & & & & & 09 & 56 & 53.57 \\
\hline & & & 10 & 32 & 58.56 & & & & & 10 & 43 & 09.56 \\
\hline & & & 11 & 19 & 17.54 & & & & & 11 & 29 & 27.54 \\
\hline
\end{tabular}

Table 18. TRMM and Parabola are Visible During the Following Time Periods

\begin{tabular}{|c|c|c|c|c|c|c|c|c|c|c|c|c|}
\hline \multicolumn{3}{|c|}{ Date } & \multicolumn{3}{|c|}{ Time } & \multirow[b]{2}{*}{ To } & \multicolumn{3}{|c|}{ Date } & \multicolumn{3}{|c|}{ Time } \\
\hline$\underset{\nabla}{\grave{\Xi}}$ & $\begin{array}{l}\bar{\Xi} \\
\dot{\bar{z}}\end{array}$ & $\vec{\Xi}$ & $\stackrel{\Xi}{\Xi}$ & $\stackrel{\Xi}{\Xi}$ & 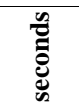 & & $\underset{\nu}{\grave{J}}$ & $\begin{array}{l}\tilde{E} \\
\bar{\Xi}\end{array}$ & $\stackrel{\vec{\sigma}}{\theta}$ & $\grave{\Xi}$ & $\stackrel{\Xi}{E}$ & 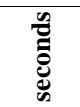 \\
\hline & & & 12 & 54 & 13.98 & \multirow{3}{*}{ to } & & & & 13 & 04 & 23.98 \\
\hline & & & 13 & 40 & 29.97 & & & & & 13 & 50 & 39.96 \\
\hline & & & 15 & 13 & 02.94 & & & & & 15 & 23 & 12.93 \\
\hline
\end{tabular}


Table 18. cont...

\begin{tabular}{|c|c|c|c|c|c|c|c|c|c|c|c|c|}
\hline \multicolumn{3}{|c|}{ Date } & \multicolumn{3}{|c|}{ Time } & \multirow[b]{2}{*}{ To } & \multicolumn{3}{|c|}{ Date } & \multicolumn{3}{|c|}{ Time } \\
\hline$\stackrel{\Xi}{\beth}$ & 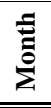 & $\vec{\Xi}$ & 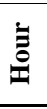 & $\underset{\Xi}{\stackrel{\Xi}{\Xi}}$ & 葛 & & 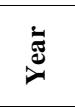 & 忢 & ڤి & $\stackrel{\Xi}{\Xi}$ & $\stackrel{\Xi}{\stackrel{\Xi}{\Xi}}$ & 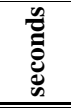 \\
\hline & & & 15 & 59 & 19.92 & & & & & 16 & 09 & 30.92 \\
\hline & & & 16 & 45 & 36.91 & & & & & 16 & 55 & 46.90 \\
\hline & & & 17 & 31 & 52.89 & & & & & 17 & 42 & 03.89 \\
\hline & & & 18 & 18 & 09.88 & & & & & 18 & 28 & 19.87 \\
\hline & & & 19 & 04 & 26.86 & & & & & 19 & 14 & 36.86 \\
\hline & & & 19 & 50 & 42.85 & & & & & 20 & 00 & 52.84 \\
\hline & & & 20 & 36 & 59.83 & & & & & 20 & 47 & 09.83 \\
\hline & & & 21 & 23 & 15.82 & & & & & 21 & 33 & 25.81 \\
\hline & & & 22 & 09 & 32.80 & & & & & 22 & 19 & 42.80 \\
\hline & & & 22 & 55 & 48.79 & & & & & 23 & 05 & 58.78 \\
\hline & & & 23 & 42 & 05.77 & & & & & 23 & 52 & 15.77 \\
\hline \multirow[t]{15}{*}{2008} & 05 & 23 & 00 & 28 & 22.76 & \multirow{15}{*}{ to } & 2008 & 05 & 23 & 00 & 38 & 31.75 \\
\hline & & & 01 & 14 & 38.74 & & & & & 01 & 24 & 49.74 \\
\hline & & & 02 & 00 & 55.73 & & & & & 02 & 11 & 05.72 \\
\hline & & & 02 & 47 & 11.71 & & & & & 02 & 57 & 22.71 \\
\hline & & & 03 & 33 & 28.70 & & & & & 03 & 43 & 38.69 \\
\hline & & & 04 & 19 & 45.68 & & & & & 04 & 29 & 55.68 \\
\hline & & & 05 & 06 & 01.67 & & & & & 05 & 16 & 11.66 \\
\hline & & & 05 & 52 & 18.65 & & & & & 06 & 2 & 28.65 \\
\hline & & & 06 & 38 & 34.64 & & & & & 06 & 48 & 44.63 \\
\hline & & & 07 & 24 & 51.62 & & & & & 07 & 35 & 01.62 \\
\hline & & & 08 & 11 & 07.61 & & & & & 08 & 21 & 17.60 \\
\hline & & & 08 & 57 & 24.59 & & & & & 09 & 07 & 34.59 \\
\hline & & & 09 & 43 & 41.58 & & & & & 09 & 53 & 50.57 \\
\hline & & & 10 & 29 & 57.56 & & & & & 10 & 40 & 08.56 \\
\hline & & & 11 & 16 & 14.55 & & & & & 11 & 26 & 24.54 \\
\hline
\end{tabular}

Table 19. GOES_3 and NOAA_3 are Visible During the Following Time Periods

\begin{tabular}{|c|c|c|c|c|c|c|c|c|c|c|c|c|}
\hline \multicolumn{3}{|c|}{ Date } & \multicolumn{3}{|c|}{ Time } & \multirow[b]{2}{*}{ To } & \multicolumn{3}{|c|}{ Date } & \multicolumn{3}{|c|}{ Time } \\
\hline$\stackrel{\Xi}{\Xi}$ & 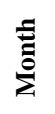 & పే & $\stackrel{\Xi}{\Xi}$ & $\stackrel{\Xi}{\Xi}$ & 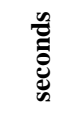 & & $\stackrel{\Xi}{\Xi}$ & $\stackrel{\Xi}{\underline{\Xi}}$ & $\vec{\theta}$ & $\stackrel{\Xi}{\Xi}$ & $\stackrel{\stackrel{\Xi}{\Xi}}{\Xi}$ & 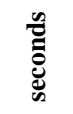 \\
\hline 2008 & 05 & 22 & 12 & 15 & 12.00 & \multirow{9}{*}{ to } & 2008 & 05 & 22 & 12 & 40 & 51.99 \\
\hline & & & 13 & 18 & 42.97 & & & & & 13 & 41 & 38.97 \\
\hline & & & 14 & 09 & 35.96 & & & & & 14 & 32 & 02.95 \\
\hline & & & 15 & 10 & 56.94 & & & & & 15 & 34 & 13.93 \\
\hline & & & 16 & 00 & 11.92 & & & & & 16 & 25 & 01.91 \\
\hline & & & 17 & 00 & 23.90 & & & & & 17 & 32 & 11.89 \\
\hline & & & 17 & 42 & 19.89 & & & & & 18 & 19 & 06.88 \\
\hline & & & 18 & 42 & 48.87 & & & & & 21 & 44 & 28.81 \\
\hline & & & 22 & 4 & 47.80 & & & & & 23 & 24 & 59.78 \\
\hline
\end{tabular}


Table 19. cont...

\begin{tabular}{|c|c|c|c|c|c|c|c|c|c|c|c|c|}
\hline \multicolumn{3}{|c|}{ Date } & \multicolumn{3}{|c|}{ Time } & \multirow[b]{2}{*}{ To } & \multicolumn{3}{|c|}{ Date } & \multicolumn{3}{|c|}{ Time } \\
\hline 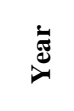 & $\stackrel{\bar{\Xi}}{\Sigma}$ & 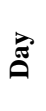 & $\stackrel{\Xi}{\Xi}$ & 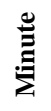 & 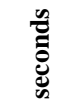 & & $\underset{\beth}{\grave{\Xi}}$ & 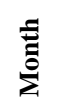 & $\overrightarrow{\tilde{\theta}}$ & 音 & $\stackrel{\Xi}{\Xi}$ & 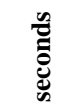 \\
\hline & & & 23 & 59 & 06.77 & & 2008 & 05 & 23 & 00 & 25 & 02.76 \\
\hline 2008 & 05 & 23 & 00 & 49 & 46.75 & & 2008 & 05 & 23 & 01 & 13 & 41.74 \\
\hline & & & 01 & 52 & 18.73 & & & & & 02 & 14 & 48.72 \\
\hline & & & 02 & 42 & 58.71 & & & & & 03 & 5 & 37.71 \\
\hline & & & 03 & 43 & 55.69 & & & & & 04 & 8 & 31.68 \\
\hline & & & 04 & 31 & 56.68 & to & & & & 04 & 58 & 58.67 \\
\hline & & & 05 & 31 & 51.66 & & & & & 06 & 53 & 32.63 \\
\hline & & & 07 & 10 & 28.63 & & & & & 10 & 12 & 53.57 \\
\hline & & & 10 & 39 & 05.56 & & & & & 11 & 12 & 42.55 \\
\hline & & & 11 & 27 & 27.54 & & & & & 11 & 56 & 45.53 \\
\hline
\end{tabular}

Table 20. NOAA_3 and Hyp_1 are Visible During the Following Time Periods

\begin{tabular}{|c|c|c|c|c|c|c|c|c|c|c|c|c|}
\hline \multicolumn{3}{|c|}{ Date } & \multicolumn{3}{|c|}{ Time } & \multirow[b]{2}{*}{ To } & \multicolumn{3}{|c|}{ Date } & \multicolumn{3}{|c|}{ Time } \\
\hline$\dot{\bar{J}}$ & 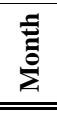 & $\stackrel{\vec{\Xi}}{\vec{I}}$ & 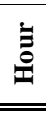 & 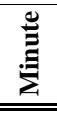 & 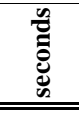 & & $\stackrel{\Xi}{\bar{J}}$ & 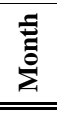 & $\vec{\theta}$ & 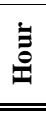 & $\stackrel{\stackrel{\Xi}{\Xi}}{. \Xi}$ & 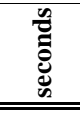 \\
\hline 2008 & 05 & 22 & 12 & 27 & 50.99 & \multirow{12}{*}{ to } & 2008 & 05 & 22 & 12 & 54 & 06.98 \\
\hline & & & 13 & 25 & 52.97 & & & & & 13 & 52 & 04.96 \\
\hline & & & 14 & 23 & 56.95 & & & & & 14 & 50 & 11.94 \\
\hline & & & 15 & 21 & 59.93 & & & & & 15 & 48 & 09.93 \\
\hline & & & 16 & 20 & 02.92 & & & & & 16 & 46 & 16.91 \\
\hline & & & 17 & 18 & 05.90 & & & & & 17 & 44 & 14.89 \\
\hline & & & 18 & 16 & 08.88 & & & & & 18 & 42 & 21.87 \\
\hline & & & 19 & 14 & 11.86 & & & & & 19 & 40 & 20.85 \\
\hline & & & 20 & 12 & 14.84 & & & & & 20 & 38 & 27.83 \\
\hline & & & 21 & 10 & 17.82 & & & & & 21 & 36 & 25.81 \\
\hline & & & 22 & 08 & 20.80 & & & & & 22 & 34 & 32.79 \\
\hline & & & 23 & 06 & 23.78 & & & & & 23 & 32 & 30.77 \\
\hline \multirow[t]{13}{*}{2008} & 05 & 23 & 00 & 04 & 26.76 & \multirow{13}{*}{ to } & 2008 & 05 & 23 & 00 & 30 & 37.76 \\
\hline & & & 01 & 02 & 30.75 & & & & & 01 & 28 & 36.74 \\
\hline & & & 02 & 00 & 33.73 & & & & & 02 & 26 & 42.72 \\
\hline & & & 02 & 58 & 36.71 & & & & & 03 & 24 & 41.70 \\
\hline & & & 03 & 56 & 39.69 & & & & & 04 & 22 & 48.68 \\
\hline & & & 04 & 54 & 42.67 & & & & & 05 & 20 & 46.66 \\
\hline & & & 05 & 52 & 45.65 & & & & & 06 & 18 & 53.64 \\
\hline & & & 06 & 50 & 48.63 & & & & & 07 & 16 & 52.62 \\
\hline & & & 07 & 48 & 51.61 & & & & & 08 & 14 & 58.60 \\
\hline & & & 08 & 46 & 54.59 & & & & & 09 & 12 & 57.59 \\
\hline & & & 09 & 44 & 57.58 & & & & & 10 & 11 & 04.57 \\
\hline & & & 10 & 43 & 01.56 & & & & & 11 & 09 & 03.55 \\
\hline & & & 11 & 41 & 03.54 & & & & & 12 & 00 & 00.00 \\
\hline
\end{tabular}


Table 21. NOAA_3 and Parabola are Visible During the Following Time Periods

\begin{tabular}{|c|c|c|c|c|c|c|c|c|c|c|c|c|}
\hline \multicolumn{3}{|c|}{ Date } & \multicolumn{3}{|c|}{ Time } & \multirow[b]{2}{*}{ To } & \multicolumn{3}{|c|}{ Date } & \multicolumn{3}{|c|}{ Time } \\
\hline 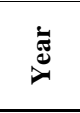 & 音 & $\vec{\theta}$ & $\stackrel{\Xi}{\Xi}$ & $\stackrel{\Xi}{\Xi}$ & 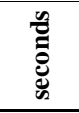 & & $\underset{\nu}{\grave{\Xi}}$ & 音 & $\vec{\Xi}$ & $\stackrel{\Xi}{\Xi}$ & $\stackrel{\Xi}{\Xi}$ & 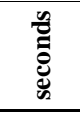 \\
\hline 2008 & 05 & 22 & 12 & 00 & 00.00 & \multirow{13}{*}{ to } & 2008 & 05 & 22 & 12 & 00 & 27.00 \\
\hline & & & 12 & 29 & 29.99 & & & & & 12 & 58 & 32.98 \\
\hline & & & 13 & 27 & 36.97 & & & & & 13 & 56 & 33.96 \\
\hline & & & 14 & 25 & 36.95 & & & & & 14 & 54 & 38.94 \\
\hline & & & 15 & 23 & 42.93 & & & & & 15 & 52 & 39.92 \\
\hline & & & 16 & 21 & 42.91 & & & & & 16 & 50 & 44.91 \\
\hline & & & 17 & 19 & 48.90 & & & & & 17 & 48 & 45.89 \\
\hline & & & 18 & 17 & 48.88 & & & & & 18 & 46 & 50.87 \\
\hline & & & 19 & 15 & 54.86 & & & & & 19 & 44 & 51.85 \\
\hline & & & 20 & 13 & 54.84 & & & & & 20 & 42 & 56.83 \\
\hline & & & 21 & 12 & 00.82 & & & & & 21 & 40 & 57.81 \\
\hline & & & 22 & 10 & 00.80 & & & & & 22 & 39 & 03.79 \\
\hline & & & 23 & 08 & 07.78 & & & & & 23 & 37 & 03.77 \\
\hline \multirow[t]{13}{*}{2008} & 05 & 23 & 00 & 06 & 06.76 & \multirow{13}{*}{ to } & 2008 & 05 & 23 & 00 & 35 & 09.75 \\
\hline & & & 01 & 04 & 13.74 & & & & & 01 & 33 & 09.74 \\
\hline & & & 02 & 02 & 13.73 & & & & & 02 & 31 & 15.72 \\
\hline & & & 03 & 00 & 19.71 & & & & & 03 & 29 & 16.70 \\
\hline & & & 03 & 58 & 19.69 & & & & & 04 & 27 & 21.68 \\
\hline & & & 04 & 56 & 25.67 & & & & & 05 & 25 & 22.66 \\
\hline & & & 05 & 54 & 25.65 & & & & & 06 & 23 & 27.64 \\
\hline & & & 06 & 52 & 31.63 & & & & & 07 & 21 & 28.62 \\
\hline & & & 07 & 50 & 31.61 & & & & & 08 & 19 & 33.60 \\
\hline & & & 08 & 48 & 37.59 & & & & & 09 & 17 & 34.58 \\
\hline & & & 09 & 46 & 37.57 & & & & & 10 & 15 & 40.57 \\
\hline & & & 10 & 44 & 44.56 & & & & & 11 & 13 & 40.55 \\
\hline & & & 11 & 42 & 43.54 & & & & & 12 & 00 & 00.00 \\
\hline
\end{tabular}

\section{ACKNOWLEDGEMENT}

None declared

\section{CONFLICTS OF INTEREST}

None declared.

\section{REFERENCES}

[1] Maini AK, Agrawal V. Satellite Technology-Principles and Applications, UK: Wiley, 2007.

[2] Noton M. Spacecraft navigation and guidance, advances in industrial control. Berlin: Springer-Verlag 1998
[3] Visser PNA, Wakker KF, Ambrosius BAC. Global gravity field recovery from the ARISTOTELES satellite mission. J Geophys Res 1994; 99(B2): 2841-51.

[4] Reinhard R, Jafry Y, Laurance R. STEP - a satellite test of the equivalence principle. ESA J 1993; 17(3): 251-63.

[5] Schwintzer P, Kang Z, Reigber C, Zhu SY. GPS satellite-tosatellite tracking for TOPEX/Poseidon precise orbit determination and gravity field model improvement. J Geodyn 1995; 20(2): 15566.

[6] Visser PNAM, Ambrosius BAC, Wakker KF. Gravity field model adjustment from ers-1 and TOPEX altimetry and slr tracking using an analytical orbit perturbation theory. Adv Space Res 1995; 16(12): 143-7.

(C) Sharaf et al.; Licensee Bentham Open.

This is an open access article licensed under the terms of the Creative Commons Attribution Non-Commercial License (http://creativecommons.org/licenses/by-nc/3.0/) which permits unrestricted, non-commercial use, distribution and reproduction in any medium, provided the work is properly cited. 